\title{
Óleo de coco, banha de porco, prazer de comer e os deslizamentos do saudável: manejos de versões da Doença de Alzheimer a partir de terapias alimentares
}

\author{
Cíntia Liara Engel ${ }^{1}$ \\ Universidade de Brasília
}

Resumo: Durante uma consulta em um ambulatório da demência do Distrito Federal, presenciei certa disputa que me fez atentar para a centralidade das práticas alimentares para a Doença de Alzheimer. A partir de desdobramentos dessa consulta e posteriores entrevistas e observações realizadas entre 2016 e 2018 - ao longo de uma pesquisa etnográfica sobre as terapias da Doença de Alzheimer -, reflito sobre disputas e testagens de alimentos como tipo de terapia; as quais, concluo, fazem parte central nas produções de distintas versões acerca do que é a doença e do que é saúde e corpo nesse contexto.

Palavras-chave: Doença de Alzheimer, alimentação, corpo e saúde. 


\title{
Coconut oil, lard, pleasure of eating and the slips of the healthy: management of versions of Alzheimer's Disease from food therapies
}

\begin{abstract}
During a consultation at a Federal District/Brazil dementia outpatient clinic, I witnessed a dispute that made me aware of the centrality of eating practices for Alzheimer's Disease. From this consultation and subsequent interviews and observations conducted between 2016 and 2018 - along an ethnographic research on therapies of Alzheimer's disease - I reflect on disputes and food testing as a type of therapy; which, I conclude, are central to the productions of different versions of what is the disease and what is health and body in this context.
\end{abstract}

Keywords: Alzheimer's disease, diet, body and health.

\section{El aceite de coco, la manteca de cerdo, el placer de comer y los deslices de lo saludable: manejo de las versiones de la enfermedad de Alzheimer de terapias alimentarias}

\begin{abstract}
Resumen: Durante una consulta en una clínica ambulatoria de demencia en el Distrito Federal/Brasil, presencié una cierta disputa que me hizo prestar atención a la importancia de las prácticas alimentarias para la enfermedad de Alzheimer. A partir de esta consulta y entrevistas y observaciones posteriores realizadas entre 2016 y 2018, a través de una investigación etnográfica sobre terapias de la enfermedad de Alzheimer, reflexiono sobre disputas y pruebas con alimentos como un tipo de terapia; que, concluyo, son fundamentales para la producción de diferentes versiones de lo que es la enfermedad y lo que es la salud y el cuerpo en este contexto.
\end{abstract}

Palabras clave: enfermedad de Alzheimer, dieta, cuerpo y salud. 
$\mathrm{A}$ Doença de Alzheimer é incluída entre as chamadas demências. Grosseiramente, a doença é caracterizada por afetar - de modo progressivo - as funções cognitivas: como memória, humor, fala e percepção. Apesar do sucesso político, epidemiológico e biomédico que as redes e o diagnóstico da Doença de Alzheimer ganharam no último século, se transformando em matéria de interesse coletivo, o seu diagnóstico, tratamento e definição são temas de debates acalorados, pelo menos desde 1910 (FOX, 1989; COHEN, 1998; BALLENGER, 2006; 2009; LOCK, 2013).

Produzem-se, constantemente, diferentes versões do que vem a ser a doença (KITWOOD, 1997; MOSER, 2008; LOCK, 2013), a patologia (FOX, 1989; LOCK, 2013) e as estruturas de cuidado necessárias (O'DONNOVAN et al., 2013; JENKINS, 2014). Ademais, diferentes profissionais e circuitos apresentam alternativas sobre como intervir por meio de substâncias - entre elas ingredientes, medicamentos alopáticos e vitaminas (BALLENGER et al., 2009; COHEN, 1998; LEIBING; 2009a; 2014). Nenhum dos tratamentos, pelo menos de maneira ampla e acessível, promete a cura da doença, mas eles são utilizados para retardar o desenvolvimento progressivo de suas fases, divididas entre: leve, moderada e grave; ou ainda para abrandar alguns sintomas cotidianos: como humores indesejados, delírios e esquecimentos (BALLENGER et al., 2009).

Desse modo, a Doença de Alzheimer é, ao mesmo tempo, matéria de interesse coletivo amplamente partilhada, assim como objeto de disputa - o que cria intervenções, circulação de substâncias e políticas de natureza variadas, por vezes conflitantes (MOSER, 2006). Dentre as muitas versões produzidas sobre a Doença de Alzheimer, corpo e saúde deslizam e são conceituados de modos particulares: a patologia reconhecida pode não ter relação com o princípio ativo do medicamento; o medicamento pode gerar efeitos difusos; a garantia da qualidade de vida pode envolver tanto diminuir a quantidade de medicamentos como aumentá-la; a saúde pode significar a longevidade, ou, no lugar disso, a paz doméstica.

Tal complexidade do universo da Doença de Alzheimer e suas intervenções têm sido abordadas pela antropologia da saúde e da ciência e tecnologia (FOX, 1989; LEIBING, 1999; MOSER, 2008; LOCK, 2013; BALLENGER et al., 2009), mas raramente por meio da valoração, escolha e manipulação de ingredientes e do cuidado alimentar, com algumas exceções. Para Habers, Mol e Stollmeyer (2002) e Mol (2010), os modos como a comida é manejada no cotidiano de cuidado são centrais para compreender o que é considerado uma boa prática e quais bens estão em jogo. As autoras sugerem que focar nas práticas alimentares é uma estratégia privilegiada de se aproximar de como terapias, doença e corpo são compostas (enact) em contextos específicos.

Seguindo as autoras, Brijanaht (2011) argumenta que as escolhas e manipulações de ingredientes ocupam bastante tempo nas lidas cotidianas com a demência. Para Brijanaht, tal processo não é unívoco e pode perpassar diferentes intenções. Alguns ingredientes, como o doce, podem ser consumidos para aproximarse das pessoas com demência e melhorar a interação, já outros são utilizados pelo 
seu valor de intervenção no humor, no sono, nas incontinências e evacuações diárias. $\mathrm{O}$ cuidado com a alimentação, então, envolve diferentes modos de abordar e conviver com a doença no cotidiano.

Neste artigo, argumento junto com essas autoras sobre a oportunidade privilegiada de trazer as práticas sociomateriais de manejo da comida para o centro dos relatos sobre o cuidado, a demência e suas lógicas. Meu objetivo principal, contudo, não é chamar atenção para como tais práticas são centrais ao cuidado cotidiano, mas seguir, a partir delas, determinadas perspectivas sobre o que vem a ser a doença, como, o quê e porquê tratar. Para tanto, começo o texto seguindo uma divergência sobre o óleo de coco, um ingrediente que agregou, em meu campo, uma série de posições sobre como tratar a demência. Esse ingrediente me leva a versões da demência, de corpo e do que se busca como terapia que o excedem e o conectam com determinados circuitos. Nos relacionaremos, neste texto, com a movimentação de familiares, a geriatria e a gerontologia e a urbanização dos cuidados e comidas - os quais não encerram, de forma alguma, os muitos circuitos que disputam as terapias da Doença de Alzheimer.

Seguindo Moser (2008), acredito que as produções de importância acerca de doenças e intervenções biomédicas mobilizam-se em muitos locais, para além do consultório, ou das pesquisas laboratoriais. $\mathrm{O}$ autor sugere que, ao se preocupar com as políticas de natureza biomédica, é importante trazer ao debate distintos lugares e versões das 'doenças' e seus circuitos. O autor argumenta, inspirandose em Butler (1990) e Mol (2002), que realidades materiais não são estáveis, ou fechadas, mas dependem de um constante processo de feitura (enact) por meio de práticas situadas. Vou adotar esse conceito para pensar sobre os debates de determinados ingredientes e as versões da demência compostas e tensionadas.

Os dados desse artigo se referem a uma pesquisa etnográfica que fiz para o meu doutorado entre os anos de 2016 e 2018 sobre as práticas de cuidado, lidas com remédios e outras intervenções terapêuticas das demências e Doença de Alzheimer. Observei consultas de uma geriatria vinculada à rede pública de saúde do Distrito Federal. Li e consultei prontuários. E, finalmente, convivi e me inseri nos cotidianos de cuidado de três famílias. De modo complementar, realizei algumas entrevistas, acumulei fotos de materiais de cuidado e visitei locais relevantes para o cotidiano - como farmácias e supermercados ${ }^{2}$.

O artigo é dividido em quatro seções etnográficas, mais as considerações finais. Primeiro, apresento um dia de consulta, no qual o óleo de coco se transformou em objeto de debate de uma filha, uma geriatra e uma cuidadora profissional. Nesse dia, conheci algumas versões da demência e notei que seguir práticas alimentares e escolhas de ingredientes poderia articular coisas importantes nas composições e manejos da Doença de Alzheimer. Nas três seções seguintes, acompanho as versões que atravessaram esse encontro: a da filha e de familiares engajados na busca por terapias; a da geriatria multidisciplinar que conheci; a de uma profissional de cuidado terceirizada.

${ }^{2}$ Agradeço ao Conselho Nacional de Desenvolvimento Científico e Tecnológico (CNPq) pelo financiamento do desenvolvimento do projeto e da pesquisa de doutorado. E agradeço à Annette Leibing por me incluir no projeto coordenado por ela, A terceira onda da demência: uma etnografia da mudança no Brasil, e financiado pela Universidade de Montreal (UdeM). Alguns dos dados desse artigo e da pesquisa de doutorado - aqueles coletados no centro de medicina - fazem parte do escopo desse projeto. 


\section{Um dia de consulta: a divergência}

O centro de medicina no qual fiz a pesquisa é vinculado ao Sistema Único de Saúde (SUS) do Distrito Federal. Trata-se de um centro especializado em geriatria dentro de um Hospital Universitário, atende pessoas que foram encaminhadas pelas Unidades Básicas de Saúde ou por médicos de outras especialidades (sejam eles profissionais da rede pública ou privada). A triagem e o acolhimento do serviço recebem as pessoas encaminhadas, investigam e categorizam possíveis condições de saúde. O centro é, também, uma referência para o diagnóstico da Doença de Alzheimer, muitos dos encaminhados carregam consigo a suspeita dessa doença. Depois de montados os diagnósticos, as pessoas podem ser encaminhadas para três ambulatórios: o da psiquiatria geriátrica, da geriatria geral ou das demências.

Muitas pessoas chegam ao centro com mais de uma condição de saúde, tomam diferentes medicamentos e são categorizadas, assim, como "casos complexos”. Muitas demências, nesse contexto, são também avaliadas como casos complexos que contém diagnósticos como: Doença de Alzheimer, demência mista, Parkinson ou demência vascular - mas não só, já que podem incluir hipertensão, diabetes, osteoporose, insuficiência cardíaca e outros. A metodologia dessa geriatria, que é formada por uma equipe multidisciplinar, envolve uma avaliação relativamente ampla do contexto geral de sintomas, experimentações com medicamentos, relações e cotidianos de cuidado; e as consultas tocam todos esses temas. São, assim, longas, densas e cheias de idas e vindas.

Logo no início de minhas observações nos ambientes de consulta, acompanhei o atendimento de uma família no ambulatório da demência. $\mathrm{O}$ caso era antigo, o que podia ser percebido pelo tamanho do prontuário e pelo tempo tomado pela médica no começo da consulta para folheá-lo e iniciar a conversa a partir de informações já anotadas. Tal prontuário, como vim saber mais adiante, contém informações das mais diversas, desde relatos biográficos a testes de memória, nomes de doenças, lista remédios utilizados, resultados de exames, anotações sobre dilemas enfrentados no cuidado cotidiano, alimentação, incontinências...

Era D. Carmela ${ }^{3}$ quem estava sendo atendida - uma mulher de quase 80 anos, diagnosticada há sete com uma Doença de Alzheimer. Além dela, estavam na sala de consulta a filha Marília, a cuidadora contratada Carla, a médica Dra. Lara e eu. Dra. Lara, em algum momento da consulta - como de praxe - perguntou como estavam os hábitos alimentares de D. Carmela. Marília relatou, com preocupação, que sua mãe estava muito apegada a guloseimas e doces. Ao que D. Carmela retrucou, dizendo que não, não era de seu feitio comer doces.

Marília deixou a mãe falar, mexeu a cabeça em negativa para comunicar à médica que não era verdade e passou a falar sobre as mudanças que fez na dieta da mãe e como isso era central para a sua atividade de cuidado. Contou que havia, há mais ou menos quatro meses, mudado o cardápio da casa, porque queria, por meio do consumo de vitaminas B12 e ômega 3 (conhecidas como boas para a memória e raciocínio), que a mãe melhorasse um pouco a sua situação. Por esse mesmo motivo, trocou todo o consumo de óleo da casa para o óleo de coco - pois soube que tal alimento teria um resultado terapêutico para a demência.

Marília aproveitou o espaço do consultório para comentar com Carla, a cuidadora, que o óleo de coco estava acabando muito rápido e que, talvez, isso estava ocorrendo porque ela utilizava mais do que o necessário na hora de fazer a comida e, assim, seria bom controlar, porque o produto é custoso para a renda mensal. 
Carla disse que usava "só o normal”, não teria como usar menos. Marília insistiu um pouco, adicionando ainda que era importante variar mais no cardápio do almoço, diminuindo a quantidade de carboidratos e aumentando a de legumes; e controlar as guloseimas que Carmela pedia. "É importante cuidar da alimentação, né, doutora?", perguntou Carla.

Dra. Lara respondeu, mediando o diálogo das duas, que mudar a dieta inteira nessa altura do campeonato talvez não fizesse mais muita diferença. Os efeitos positivos de uma dieta com pouco carboidrato e rica em vitaminas seriam mais evidentes depois de anos de consumo. Com quase 80 anos, D. Carmela talvez não se beneficiasse. Disse ainda que ela poderia comer algumas guloseimas, já que não tinha diabetes. E, já em fase moderada da demência, Dra. Lara falou que o seu medo era que, em algum momento, Carmela parasse de comer. O importante, nesse caso, era comer e segurar o peso: "porque o peso é um fator de proteção nos idosos”. Mas, vendo que Marília não gostou do comentário, acrescentou que era muito bom variar o cardápio e incluir coisas saudáveis.

A consulta seguiu, as desavenças sobre a alimentação foram deixadas de lado para que outros assuntos fossem abordados. Nesse e em muitos outros momentos da pesquisa, a decisão sobre o que e como comer ganhou centralidade. E ela, como fui perceber, envolvia disputas intensas sobre corpo, demência e saúde. Sem dúvida, isso se deve ao reconhecido papel que as práticas alimentares têm no cuidado cotidiano, especialmente nos casos de demência (HABERS, MOL e STOLLMEYER, 2002), assim como nas diferentes intenções sobre as práticas alimentares, sejam elas de intervenção em determinados sintomas, de prazer culinário, de garantia de nutrição ou de manutenção de vínculos afetivos (BRIJANAHT, 2011). Sigo o artigo argumentando, ainda, que os manejos e decisões sobre as práticas alimentares podem também nos aproximar de diferentes versões das demências, das decorrentes estratégias, disputas e acúmulos de práticas terapêuticas e das muitas relações que compõem e são compostas pelos seus circuitos de cuidado.

\section{Explorar todas as alternativas - versões da demência e o óleo de coco}

Depois de presenciar essa consulta, convidei Marília para uma entrevista. A recebi em minha casa, por decisão dela. Marília me contou muitas coisas sobre a história da doença de sua mãe e sobre a sua abordagem de cuidado. Marília desconfiava muito do tratamento medicamentoso para as demências. Disse-me que ouviu dos médicos e leu em suas pesquisas em livros e internet que tais medicamentos eram limitados e que, no máximo, poderiam segurar o avanço da doença por alguns anos. Não era um motivo suficiente, em sua opinião, para não os utilizar, já que "pode ser que eles ajudem um pouco, a gente nunca tem como saber". Mas essa abordagem, além de causar várias complicações para o cotidiano de cuidado - já que eram custosos e precisavam de muitos cuidados e atenções - não era a única possível, ou suficiente, me disse.

Marília não está sozinha nessa desconfiança. Os principais medicamentos para a Doença de Alzheimer são os anticolinesterásicos, os quais aumentam a quantidade do neurotransmissor acetilcolina no cérebro. O medicamento não se relaciona com as causas ou a patologia da doença, é inócuo em boa parte dos casos e, além disso, pode gerar vários efeitos adversos. Por isso, há um debate intenso sobre se ele é ou não positivo (BALLENGER et al., 2009). O aumento da quanti- 
dade do neurotransmissor, contudo, pode melhorar a atenção, memória e, eventualmente, o humor (ANDERSON, 2009). A memantina é outro medicamento específico para as demências, utilizado para evitar a morte de neurônios. De acordo com alguns de meus interlocutores, ele é mais indicado para a fase grave da doença, mas outros deles defendem que esse medicamento deve ser utilizado em conjunto com os anticolinesterásicos para potencializar o efeito dos dois. Muitos de meus interlocutores me disseram que seu efeito é discreto, desconfiando se é ou não bom mantê-lo, outros acreditam que mesmo um efeito discreto é positivo.

Outros medicamentos frequentemente utilizados são os antidepressivos e antipsicóticos - que manejariam humores e comportamentos (LEIBING, 2009a). O cálculo sobre utilizar ou não tais medicamentos depende de uma série de decisões e de um ajuste constante dos riscos e intenções terapêuticas, efeitos adversos, de longo prazo, indesejados, vindos da polifarmácia e do preço excessivo do conjunto medicamentoso (LEIBING, ENGEL e CARRIJO, 2019).

Foi Marília quem retomou a consulta, me dizendo "Eu não gostei muito do que a médica falou, que não ia fazer diferença mexer na alimentação deles". Disse-me que sabia que sua mãe estava conseguindo manter muita coisa na sua vida cotidiana e que isso se relacionava com o esforço que ela fazia em gerir e criar estratégias para o cuidado. Listou as atividades que ela inseriu na rotina de D. Carmela: faz exercícios, palavras cruzadas, terapia ocupacional, coral... "eu tento tudo o que você imaginar”.

Sobre a alimentação, Marília argumentou que viu um vídeo, anos atrás, de uma médica, cujo marido tinha Alzheimer. Essa médica havia iniciado, por conta própria, um tratamento baseado no óleo de coco e o marido melhorou. $\mathrm{O}$ fato, inclusive, foi medido pelos mesmos testes de progressão da doença utilizados pelo centro - como um que envolve desenhar um relógio. Marília mexeu em sua bolsa, pegou o celular, procurou o vídeo e me mandou ele pelo WhatsApp. Continuou me dizendo que essa história virou um livro, um best seller e inspirou várias pesquisas. "Eu faço tudo o que eu posso e essa parte da alimentação me dá muito trabalho. Eu penso no cardápio, compro, penso nas receitas. E eu sei que ela tá bem não é só por causa dos remédios, é por causa dessas outras coisas que eu faço, inclusive a alimentação”. Isso não era barato, me disse, especialmente porque a alimentação da casa era também consumida pela cuidadora e pelo marido de D. Carmela. O óleo de coco, assim, acabava sendo um peso. Marília novamente afirmou que achava difícil dialogar com Carla sobre as medidas e o uso adequado do produto, o que a estava fazendo repensar essa relação de trabalho, apesar de Carla, em sua opinião, ser ótima em outras atividades de cuidado.

Tive outras oportunidades de conversar e conviver com Marília. Ela me indicou muitos livros sobre alternativas de terapia do Alzheimer e outras demências, me falou sobre a rede de instituições e possibilidades de cuidado no Distrito Federal (DF) e partilhou comigo o seu desejo de criar espaços mais adequados para a convivência de pessoas com demência. Marília pesquisava muito sobre a doença da mãe e criava estratégias e terapias a partir disso. Era importante para ela tentar todas as alternativas que pudesse.

Quando Marília saiu daquela entrevista, assisti ao vídeo. Em 2008, a médica cuja experiência Marília havia me indicado pesquisar - a americana Mary Newport - estava frustrada com a piora de seu marido, diagnosticado com a Doença de Alzheimer. Na ida para uma entrevista que incluiria (ou não) o marido em um estudo para a testagem de um novo medicamento, ouviu uma notícia sobre a metabolização deficiente de glicose pelo cérebro em pessoas com o diagnóstico da 
Doença de Alzheimer e outras demências. Tal problema poderia ser resolvido, ou pelo menos enfrentado, com o consumo de um outro tipo de fonte de energia ketones. E um dos alimentos que proporcionaria essa fonte de energia era o óleo de coco. Steve Newport, o marido de Mary, não passou na tal entrevista. Os testes tiveram resultados inferiores ao corte desejado. Um desses era a tentativa de desenho de um relógio - que ficou bem distante do que era considerado esperado para a fase leve da doença (objeto da pesquisa).

Frustrada e assustada com os resultados, Mary comprou o óleo de coco e mediu uma dosagem diária suficiente (consideravelmente alta) para intervir na situação de seu marido. Ela observou uma melhora intensa, inclusive nos mesmos testes. Passou a relatar o caso na internet, a partir de um site ${ }^{4}$ próprio, até ganhar maior visibilidade. Com o tempo, conseguiu fazer parcerias com pesquisadores que defendiam essa hipótese, criando um circuito de atenção cada vez maior para essa versão da Doença de Alzheimer e o seu possível tratamento. Mary chegou a escrever um artigo em parceria com alguns desses pesquisadores contando o "estudo de caso" feito com seu marido: suas idas e vindas, a inserção de um componente mais puro de fonte da substância e, curiosamente, a entrada do marido no estudo para a testagem do novo medicamento - já que os resultados dos testes melhoraram consideravelmente. Este estudo, contudo, não foi positivo para o casal, evidenciando ainda mais que a causa da melhora foi a nova fonte de energia para o cérebro.

Mary acumulou relatos de pessoas que fizeram os mesmos testes e publicou alguns livros (NEWPORT, 2013; 2015; 2019); deu entrevistas eternizadas em vídeos 5 e ficou consideravelmente conhecida. Hoje essa versão da demência é reconhecida em alguma medida e testes controlados estão sendo feitos para produzir intervenções 'oficiais'. Contudo, Mary também é uma figura controversa, causando a desconfiança de uma parte dos pesquisadores e médicos. Os regimes de conhecimento e buscas por cura e cuidado na Doença de Alzheimer e outras demências parecem articular, como argumentam Moreira e Palladino (2005), a produção e fortalecimento de verdades reconhecidas e institucionalizadas, ao mesmo tempo em que manejam e incluem muitas fontes alternativas de esperança.

A história de parentes de pessoas diagnosticadas com a Doença de Alzheimer que, de alguma maneira, conseguem se articular com redes maiores - sejam de médicos, pesquisadores, advogados, políticos - é longa e tem um papel central na transformação dessa doença em um tema de interesse público (FOX, 1989; BEARD, 2004; O'DONOVAN, et al., 2013). Mary se insere, assim, em um conjunto de parentes engajados na busca por pesquisa, cura e cuidado para além do que há no mercado e do conjunto de medicamentos existentes.

Essa versão da Doença de Alzheimer difere da dos medicamentos mais conhecidos - que lidam com neurotransmissores -, mas não se contrapõe à mesma. Outras versões e investimentos em pesquisa estão em curso, existem desde procuras por marcadores genéticos que possam, quem sabe, garantir um diagnóstico preventivo; tentativas de destruir as placas senis e emaranhados neurofibrilares (patologias reconhecidas da doença, apesar de também controversas), entre muitas outras (BALLENGER et al., 2009; LOCK, 2013). Tais versões nem sempre se negam entre si, mas suportam abordagens de tratamento distintas. Nenhuma dessas alternativas, contudo, era acessível para o consumo geral da prática assistencial - pelo menos não no SUS brasileiro quando fiz a pesquisa. Os remédios, contudo, eram financiados. Além disso, abordagens a partir de arte, estratégias 
específicas de cuidado, música e outras são defendidas e circuladas por pesquisadores (KONTOS, 2005; KONTOS e NAGLIE, 2009; KONTOS et al., 2010; TAYLOR, 2017; FERIANI, 2017) e, inclusive, pelo Centro que recebeu minha pesquisa. As versões parecem se acumular e depender de articulações localizadas.

Em alguma medida, Marília almeja várias possibilidades de melhoras, mesmo que pontuais, como parte de suas estratégias de cuidado. A Doença de Alzheimer não significa uma coisa só e os tratamentos acompanham esse processo - é o acúmulo e testagem de estratégias de melhoria que não façam mais mal do que bem que compõem a busca relativa por saúde, ou qualidade de vida outro termo muito importante para os tratamentos da demência. No cotidiano, Marília tenta acumular todas as informações que pesquisa e variar sua abordagem, já que nenhuma das alternativas promete, garante ou oferece uma cura final. Faz tratamento com os medicamentos, com a terapia ocupacional e com a alimentação a partir do aumento de vitaminas e da inserção do óleo de coco. Parece colocar em prática uma certa mediação de versões da demência na tentativa de manter a mãe mais atenta, tranquila e afeita a certos tipos de relação. Por isso, quando ouviu da médica que a mudança na alimentação não importava, sentiu como se uma parte fundamental do seu trabalho de pesquisa e constituição do cuidado fosse diminuída.

\section{A clínica geriátrica e a Doença de Alzheimer que, invariavelmente, piora: olhando para o conjunto}

A cena parece nos dizer que a médica não estava muito convencida de que esse tipo de intervenção alimentar faria uma diferença significativa, negando o conhecimento e as estratégias terapêuticas escolhidas pela filha. Ou ainda desconfiando de iniciativas que não tenham ganhado espaço mainstream. Essa cena caminha para essa conclusão, mas, com o tempo de pesquisa, passei a notar que ela também diz muito sobre o objeto e o tipo de cuidado e lógica de intervenção dessa geriatria multidisciplinar.

A posição dos geriatras que conheci em relação aos hábitos alimentares varia bastante. Tanto entre eles, como em relação ao caso atendido. Tenho a impressão de que tal variação se relaciona com a constituição do próprio objeto de intervenção dessa geriatria. Apesar de o ambulatório da demência dar centralidade para o cuidado dessas doenças, elas não são observadas como o único objeto de intervenção. Por diferentes caminhos, os profissionais do centro me ensinaram que a sua abordagem de cuidado e intervenção se relaciona com o conjunto - categoria que agrega a doença. Como um tipo de especialidade generalista (e multidisciplinar), focada no envelhecimento - algo parecido com a pediatria -, os geriatras incluem as demências em um conjunto de situações enfrentadas pelas pessoas envelhecidas e pelas famílias que realizam processos de cuidado e mediam suas intervenções a partir disso. A memória, as emoções, o cérebro e suas células são cuidados, mas são relacionados a um contexto maior: o organismo, o conjunto de doenças, a família, as relações de cuidado, a velhice na sociedade moderna.

Ao compor um objeto de intervenção - um caso - os geriatras parecem investigar, a partir de categorias específicas, o que pode interferir nos sintomas e na sua intervenção: sejam as relações familiares, os medicamentos utilizados ou as doenças acumuladas. Para Dra. Lara, D. Carmela não precisaria se preocupar tanto com as guloseimas. Levando isso para o conjunto, talvez esse julgamento se 
relacionasse à sua avaliação de que dentre as doenças que D. Carmela experimentava, a diabetes não era uma delas, ou mesmo o peso e o excesso de alguma taxa relacionada à alimentação. Ou, ainda, que a alimentação estava já balanceada o suficiente para o que era entendido como saudável nutricionalmente.

Ainda me parece que Dra. Lara estava colocando em prática um aprendizado sobre o cuidado da Doença de Alzheimer manejado naquele contexto (que é um espaço de formação de residentes também): não há cura; nesse caso concreto vai piorar; é preciso pensar no futuro. Certa feita, acompanhei uma cena parecida com essa, uma senhora estava perdendo muito peso, de uma hora para outra. Ela se recusava a comer, ou comia muito pouco. O geriatra olhou para mim e disse "olha, é por isso que a gente fala que o peso é um fator de proteção, quando chega nessa fase, come só o que quer". Outra vez, ouvi uma filha perguntando se estava tudo bem a mãe comer massa e arroz ao mesmo tempo, porque viu em um programa de televisão que não podia misturar os dois. A geriatra respondeu: "Assim, não é bom em geral, mas pra ela, o importante mesmo é que ela está comendo". Ou outra vez, ainda, quando um filho tentava convencer o pai a parar de comer feijoada, por causa da pressão alta, o médico adicionou: "é, tem que cuidar dessa pressão, mas dá pra diminuir a quantidade né, ou colocar menos sal, só não vai deixar ele sem comer o que ele gosta que depois isso dá problema!"

A prevenção por meio de uma alimentação saudável, que diminua os riscos de pressão alta, diabetes, obesidade e outros "fatores de risco da demência", prevista em outros contextos - e, inclusive, narrativa intensa no debate sobre o que é tratar demência contemporaneamente (LEIBING e KAMPF, 2013) -, entra em conflito com a demência que já existe, já piorou e que, dificilmente, vai conseguir, a partir do Brasil, do SUS (ou mesmo da rede privada financiada por uma família de classe média alta) acessar os novos tratamentos divulgados constantemente. A piora esperada, então, deixa a prevenção e a alimentação saudável em suspenso - a ser avaliada caso a caso.

Até o peso pode virar tema a ser discutido. O peso como fator de proteção pode significar que pessoas magras sejam aquelas que se tornem mais fragilizadas em um momento de perda do apetite, ou da capacidade mesma de mastigar e deglutir. A perda de peso rápida e exagerada intensificaria a vulnerabilidade de corpos que não possuem peso suficiente (e Dona Carmela era uma mulher bastante magra). Julgamento, contudo, que não negava a busca por uma alimentação saudável, aproximando-se e distanciando-se desse objetivo a depender do conjunto.

Havia um cálculo entre como lidar com a alimentação saudável, preventiva e necessária para controlar a pressão e diabetes, extremamente comuns, e duas máximas que pareciam também orientar o serviço: "o importante é comer" e "o peso é um fator de proteção para idosos”. Cálculo que diferenciava, ainda, o que é saudável para o corpo adulto e o que é saudável para o corpo idoso, especialmente aquele que já tem demência. Nas versões agregadas por essa geriatria, as fases avançadas da doença envolveriam a perda de apetite, vontade de comer e, então, comer por si só era importante. Mesmo que fosse só o que se gosta. Assim, o cálculo envolvia mais do que o 'saudável', mas também os processos de piora prevista e a mediação de mais de uma doença. Tal processo tornava a prática mais difícil de ser padronizada, já que é dependente de cada caso. E, nesse sentido, o conjunto entra em ação: ao olhar para esse conjunto, decide-se o que é bom em termos alimentares.

Para além desses cálculos, como um dos focos da atuação é, também, estabilizar as relações, Dra. Lara pode ter julgado um possível conflito nas relações de 
cuidado causado pelas decisões sobre a alimentação e, ao avaliar que isso poderia atrapalhar o cotidiano, tentou diminuir a importância de manter a dieta. As relações de cuidado, conflitos e disputas entram na avaliação do caso - inclusive como um dos possíveis sintomas relacionados ao desequilíbrio do conjunto. Tal versão da demência envolve a avaliação dos médicos sobre a qualidade das relações de cuidado. A saúde, aqui, desloca-se apenas da manutenção da memória, mas é voltada para as próprias relações. O corpo, os nutrientes e a demência são agregados em um conjunto maior. E, nesse caso, o conflito e a desestabilização das relações de cuidado superaram, na perspectiva dessa médica, a intervenção por meio do óleo de coco. Essa decisão poderia tomar um caminho bem diferente caso as taxas fossem outras, as doenças fossem outras, as relações fossem outras, o médico fosse outro.

Tal lida com alimentos não se alinha, necessariamente, com a esperança de melhora da memória a partir de intervenções - sejam elas alimentares ou medicamentosas. De maneira geral, os geriatras que conheci não parecem trabalhar com a esperança de melhora, mas com a estabilização contínua de humores, memórias, nutrição e relações de cuidado e, ainda, com a diminuição dos danos da piora. Saúde e terapia estão circunscritas a esse contexto. Essa versão das demências pode ser difícil de dialogar com as dos livros, pesquisas, laboratórios e propagandas sobre as alternativas que, mesmo liminarmente, manejam melhoras e promessas para futuras curas e potenciais prevenções. Ou até com a experiência de cuidado de muitos familiares que buscam, constantemente, melhorias por experiências das mais diversas, dialogando ou não com a biomedicina diretamente praticada pelos seus médicos.

\section{Doença de Alzheimer, mercado do cuidado e o orçamento doméstico no preparo da comida}

Quando conversei com a cuidadora de D. Carmela, já não era mais Carla. Uma outra mulher, um pouco mais velha, Aureliana, havia ocupado seu lugar. As duas trabalhavam como funcionárias terceirizadas, contratadas a partir de uma empresa especializada em cuidadoras de idosos - uma categoria trabalhista que acompanha a transformação do envelhecimento populacional em uma questão pública. Tais empresas têm crescido consideravelmente como opção para lidar com o cuidado de idosos sem passar diretamente pela legislação trabalhista uma nova roupagem de uma histórica precarização do emprego doméstico e de cuidado no Brasil (AZERÊDO; 1989; PINHEIRO, GONZALEZ e FONTOURA, 2012; SAFIOTTI, 1982; SORJ e FONTES, 2012; DEBERT e OLIVEIRA, 2015). As cuidadoras contratadas por essa empresa terceirizada que conheci ganhavam um valor diário próximo a $\mathbf{R} \$ 75,00$ e não possuíam direitos trabalhistas: como férias, décimo terceiro ou direito a ausência por doença. Apenas dias trabalhados eram pagos. As circulações e mudanças de funcionárias eram facilitadas nesse contexto.

Não soube com detalhes o que aconteceu para que Carla fosse demitida, mas os conflitos entre ela e Marília envolvendo o tipo de comida feita e a quantidade do óleo de coco utilizada foram centrais nessa decisão. As práticas de feitura da comida superaram a avaliação de que o resto do cuidado era bom, o que chama atenção para a importância delas nas relações de trabalho e expectativas sobre o cuidado (MOL, 2010). Aureliana disse-me que achava que a demissão aconteceu por conta da "mesmice" da comida feita por Carla: variar o cardápio foi uma das 
orientações dadas a ela assim que assumiu o posto de trabalho.

Aureliana me contou que adorava cozinhar, entre as suas funções, era a preferida. Ela era responsável por muitas coisas, entre elas a supervisão do banho, a feitura e garantia de consumo de alimentos, atividades diárias: como exercícios, música e passeios pela quadra; troca de roupas, conversas extensas, convencimento sobre o consumo de remédios e comida e a lida com sentimentos, incômodos e desejos. Com a comida, Aureliana tentava inovar e fazer coisas gostosas, porque, em sua opinião, "comer é a principal parte do dia". Aureliana ficava um pouco frustrada, contudo, porque tinha mais de 15 anos que ela não podia cozinhar como queria, fazendo receitas deliciosas, quitutes, "coisas supérfluas, sabe? Hoje em dia cada casa é uma coisa, um não pode glúten, outro sal, é bem hospitalar a comida que eu faço". As restrições alimentares diminuíam um pouco o prazer de Aureliana em cozinhar para os outros, especialmente com os ingredientes com os quais aprendeu a lidar ao longo dos anos. Ela se frustrava por não poder se engajar com a comida unicamente a partir do gosto, isso porque precisava incluir outros sentidos na alimentação, mediar desejos e muitas expectativas sobre o que era saudável. Em sua versão do cuidado, o gosto precisava ser comprometido para cuidar da demência, da pressão alta, da diabetes (assim como no contexto observado por Mol [2010] e Fleischer [2018]), mas, mesmo assim, a sua tentativa era de adaptação e de variação de cardápios, para continuar buscando o seu objetivo.

Tentei puxar novamente o assunto do óleo de coco. Aureliana estava um pouco desconfiada, apesar de eu ter participado - quando estava fazendo observações diretas no cotidiano da casa - de algumas conversas de corredor e estratégias adotadas para fazê-lo render mais e evitar os conflitos. Mas dessa vez estávamos gravando e isso deixou Aureliana mais reservada sobre o que falar. Desconversou um pouco e me disse que, em sua opinião, o óleo de coco não era a melhor alternativa. Não pelo gosto ou pela falta de potencial terapêutico, mas pelo peso que representava no orçamento doméstico e pela proximidade dele de outros alimentos industrializados. O preço de um pote de $500 \mathrm{ml}$ poderia custar $\mathrm{R} \$ 30,00$ e esse valor não era razoável para um item de cozinha fundamental, como o óleo. Ele era mais natural, concordou, mas estava desconfiada da medida em que era, de fato, natural. Em sua opinião, era tão industrializado quanto os outros. E, além disso, tinha ouvido falar que ele nem era tão bom quanto prometem: "e eles [os cientistas, imagino] mudam de opinião o tempo todo".

Aureliana cresceu na roça, usando banha de porco. Para ela, por muito tempo falaram mal desse ingrediente, mas agora estavam reconsiderando-o. Em sua opinião, a vantagem dele era ser muito mais natural, porque não industrializado. Pelo menos aquele que ela costumava consumir. Além disso, Aureliana não se lembrava de os velhos da sua região terem tantos problemas de memória, diabetes e pressão - talvez tivesse alguma relação com os produtos industrializados, conjecturou. Julgou que os produtos industrializados eram, em geral, piores para a saúde. E, por fim, me contou que conseguia comprar um quilo de banha de porco por $\mathrm{R} \$ 10,00$. Isso sim seria um preço adequado para o orçamento doméstico.

Aureliana se envolvia com a demência de D. Carmela a partir de relações de trabalho e cuidado diário. Nesse sentido, as práticas de alimentação e escolha de ingredientes também compõem (enact) políticas de mercado, relações patronais e estruturas de oferta de cuidado (BUCH, 2018). Aureliana recebe orientações e precisa cumprir com certas expectativas que, nem sempre, são manejadas somente por ela. A quebra dessas expectativas pode representar uma demissão. 
Nesse sentido, Aureliana adaptava-se, incluindo suas preferências sem causar conflitos. Claro que o termo trabalho e as relações de mercado não definem por completo as suas interações (BUCH, 2018) - como elas nunca fazem, aliás (BATISTA e ARAUJO, 2011; ZELIZER, 2011). Mas elas colocam em jogo, a partir da alimentação, versões da demência que situam as pessoas em diferentes posições dentro dos circuitos de cuidado e de elaborações sobre os alimentos e a saúde. Trazendo para a cena estratégias locais de partilha, hierarquias e divisão de tarefas dos cuidados. O que é adequado depende do quanto é saudável, mas também do quanto é viável e acessível. E, aliás, a ideia do que faz o saudável, saudável, desliza constantemente.

Aureliana julgava o óleo de coco como um ingrediente falho porque era caro. Ser caro atrapalhava uma série de aspectos do cuidado cotidiano. Era inadequado para o cotidiano de cuidado se era caro demais para a função que deveria ocupar, inacessível para boa parte das pessoas e, mesmo quando acessível, precisava ser muito controlado para não atrapalhar o orçamento doméstico. E, em seu caso, colocava as relações de trabalho em risco e intensificava dificuldades do cotidiano. A comida, nesse contexto, atravessava versões do cuidado da Doença de Alzheimer que eram marcadas por diferenças de tratamento dos ingredientes a partir de posições patronais, experiências de classe, acesso aos alimentos e hierarquias nas relações de cuidado trocadas no mercado.

Buch (2018), a partir de uma pesquisa com cuidadoras de idosos em ambiente doméstico nos EUA, entrou em contato com uma série de dilemas parecidos com esse. Observou que debates sobre o que comer, como preparar a comida, como manejar o orçamento doméstico e como mediar as relações de trabalho que se agregam às habilidades e hábitos de cuidado eram centrais para as rotinas de cuidado. Para a autora, lidar com essas questões é mais do que observar a resolução de detalhes paralelos ou burocráticos, mas participa diretamente da complexidade que é o estabelecimento de uma relação de cuidado e da busca pelo que vem a ser considerado bom e saudável ao envelhecimento. Entendo, então, que saúde, qualidade do tratamento, doença e cuidado deslizam, aqui, para o que significa manter uma casa com dinheiro e do que é, no caso das cuidadoras profissionais que conheci, trabalhar em relação a um orçamento doméstico - gerando e mediando os gastos, gostos e expectativas.

\section{Considerações finais}

A Doença de Alzheimer está em constante disputa. É uma condição complexa, variável e formada por meio de um conjunto amplo de técnicas e recursos sociomateriais. Não dei conta de todos eles, nem de uma parcela significativa. Mas resolvi seguir algumas variações relacionadas aos cuidados alimentares, que ganham menos centralidade nas narrativas sobre a doença e suas terapias. Não gostaria, com os relatos anteriores, de estabelecer necessárias conexões entre tipos de personagens e as suas versões do cuidado e da demência. Até porque, tais engajamentos podem variar consideravelmente. No lugar disso, espero ter feito aparecer maneiras a partir das quais a lida com ingredientes e cuidados da alimentação nos aproximam das muitas versões da Doença de Alzheimer e das suas composições cotidianas envolvendo corpo, saúde, mente, cérebro e políticas de cuidado. Corpo e doença, assim, são observados não pelo que são ou deixam de ser, mas pelos modos como são formulados e atravessados por determinadas práticas e suas implicações (MOL, 2002; LATOUR, 2004; MCCALLUM, ROHDEN e GRUDZINSKI, 2015). 
Com as lidas do óleo de coco, pudemos conhecer a tentativa de uma filha, Marília, de inserir na lógica de cuidados diários vários elementos e substâncias que pudessem melhorar a saúde do cérebro. A partir de sua pesquisa sobre alternativas de pesquisa não medicamentosa, essa filha me apresentou a história de Mary, quem, ao tentar melhorar a situação do marido, investiu em intervenções baseadas no consumo de um tipo de fonte de energia. Marília, Mary e tantos outros parentes engajados com movimentações biomédicas e políticas são centrais para a transformação e manutenção da Doença de Alzheimer em uma questão de interesse (FOX, 1989; MOSER, 2008; BEARD, 2004).

Conhecemos ainda como um tipo de especialidade: a geriatria multidisciplinar de um hospital universitário, lida com o cuidado da demência e insere determinadas alternativas de intervenção buscando a estabilização de seu objeto de intervenção: o conjunto - o qual modifica os termos do que é tratado na demência. É um tipo de engajamento com a doença e seus circuitos que se realiza dentro da história dessa especialidade, desse centro e do que se tenta mediar no cotidiano. A Doença de Alzheimer, apesar de central, é uma entre outras condições a serem medidas e estabilizadas - mas não necessariamente curadas. E a escolha pelo óleo de coco, ou por uma dieta muito restrita, nem sempre é coerente com a perspectiva de estabilizar o conjunto. Nem corpo nem uma doença resumem a lógica do tratamento - mas esse tal conjunto definido em seus termos.

Pelos embates e circulação do óleo de coco, ainda, pudemos conhecer estruturas de organização do cuidado a partir do mercado de trabalho de cuidadoras de idosos. E, com elas, os jogos que a alimentação, a alimentação saudável e o valor de determinados ingredientes e formas de preparo colocam em cena. O óleo de coco e o jeito de cozinhar tocam as relações de trabalho, tensões de classe e expectativas sobre os ingredientes e sobre o cuidado, trazendo ao texto outras versões da Doença de Alzheimer e suas intervenções como políticas de cuidado e trabalho mais amplas.

Tomei o debate sobre o óleo de coco, assim, como fio condutor e agente central para me aproximar dos modos pelos quais determinados conhecimentos e estratégias de intervenção e cuidado são organizados em práticas distintas e fazem com que versões da demência se enfrentem e tensionem no cotidiano. $O$ uso de um ingrediente nos conectou a manejos e deslizamentos das versões da própria doença, do corpo e da saúde.

Recebido em 14 de agosto de 2019.

Aprovado em 24 de novembro de 2019.

ANDERSON, A. "Pharmacologic treatment of dementia: a clinician's view". In.: BALLENGER, J. et al. Treating dementia: do we have a pill for it? Baltimore: The Johns Hopkins University Press, 2009. pp. 103-115.

AZERÊDO, S. "Relações entre empregadas e patroas: reflexões sobre o feminismo em países multirraciais". In: OLIVEIRA COSTA, A. de.; BRUSCHINI, M. C. 
(orgs.). Rebeldia e submissão: estudo sobre a condição feminina. São Paulo: Vértice, 1989. pp.195-220.

BALLENGER, J. "Necessary interventions: antidementia drugs and heightened expectations for aging in modern American culture”. In.: BALLENGER, J. et. al. Treating dementia: do we have a pill for it? Baltimore: The Johns Hopkins University Press, 2009. pp. 189-210.

BALLENGER, J. "The biomedical deconstruction of senility and the persistent stigmatization of old age in the United States". In.: LEIBING, A. \& COHEN, L. Thinking about dementia: culture, loss and the anthropology of senility. New Jersey: Rutgers, 2006. pp. 106-122.

BALLENGER, Jesse et al. Treating dementia: do we have a pill for it? Baltimore: The Johns Hopkins University Press, 2009.

BATISTA, A.; ARAÚJO, A. B. Intimidade e Mercado: o Cuidado de Idosos em Instituições de Longa Permanência. Sociedade e Estado, 26 (1): 25-45, 2011.

BEARD, R. Advocating voice: organizational, historical and social milieux of the Alzheimer's disease movement. Sociology of Health and Illness, 26 (6): 797-819, 2004.

BRIJNATH, B. Alzheimer's and the Indian Appetite. Medical Anthropology, 30 (6): 610-628, 2011.

BUCH, Elena. Inequalities of aging: paradoxes of independence in American Home Care. New York: New York University Press, 2018.

BUTLER, Judith. Gender Trouble: Feminism and the Subversion of Identity. New York: Routledge, 1990.

COHEN, Lawrence. No aging in India: Alzheimer's, the bad family, and other modern things. Berkeley: University of California Press, 1998.

DEBERT, G.; OLIVEIRA, A. M. A profissionalização da atividade de cuidar de idosos no Brasil. Revista Brasileira de Ciência Política, 18: 7-41, 2015.

FERIANI, Daniela. Entre sopros e assombros: Estética e experiência na doença de Alzheimer. Tese. (Doutorado em Antropologia Social), Universidade Estadual de Campinas, 2017.

FLEISCHER, Soraya. Descontrolada: Uma etnografia dos problemas de pressão. São Carlos: EdUFSCar, 2018.

FOX, P. From senility to Alzheimer Disease: the rise of the Alzheimer Disease Movement. Milbank Quarterly, 67 (58): 58-102, 1989.

HARBERS, H; MOL, A; STOLLMEYER, A. Food Matters Arguments for an Ethnography of Daily Care. Theory, Culture \& Society, 19 (5/6): 207-226, 2002.

JENKINS, N. Dementia and the inter-embodied self. Social Theory \& Health, 12 (2): 125-137, 2014.

KITWOOD, Tom. Dementia reconsidered: the person comes first. Buckinghan: Open University Press, 1997.

KONTOS, P.; NAGLIE, G. Tacit knowledge of caring and embodied selfhood. Sociology of Health \& Illness, 31 (5): 688-704, 2009.

KONTOS, P. Embodied selfhood in Alzheimer's Disease. Dementia: Sage publications, 4 (4): 553-570, 2005. 
KONTOS, P. et. al. Dementia care at the intersection of regulation and reflexivity: a critical realist perspective. Journal of Gerontology: Social Sciences, 66 (1): 119128, 2010.

LATOUR, B. How to Talk About the Body? the Normative Dimension of Science Studies. Body \& Society, 10 (2-3): 205-229, 2004.

LEIBING, A. "From the periphery to the Center: treating noncognitive, especially behavioral and psychological symptoms of dementia". In. BALLENGER, J. et. al., Treating dementia: do we have a pill for it? Baltimore: The Johns Hopkins University Press, 2009b. pp. 74-97.

LEIBING, A. Olhando para trás: os dois nascimentos da doença de Alzheimer e a senilidade no Brasil. Estudos Interdisciplinares Envelhecimento, 1: 37-56, 1999.

LEIBING, A. Tense prescriptions? Alzheimer medications and the anthropology of uncertainty. Transcultural psychiatry, 46 (1): 180-206, 2009 a.

LEIBING, A. The Earlier the Better: Alzheimer's Prevention, Early Detection, and the Quest for Pharmacological Interventions. Culture, Medicine and Psychiatry, 38 (2): 217-236, 2014.

LEIBING, A; ENGEL, C.; CARRIJO, E. Life through Medications: Dementia Care in Brazil. ReVista - Harvard Review of Latin America (Aging in the Americas Part II: facing the challenges), 2019.

LEIBING, A; KAMPF, A. Neither Body nor Brain: Comparing Preventive Attitudes to Prostate Cancer and Alzheimer's Disease. Body \& Society, 19 (4): 61-91, 2013.

LOCK, Margaret. The Alzheimer conundrum: entanglements of dementia and aging. New Jersey: Princeton University Press, 2013.

MCCALLUM, A; ROHDEN, F.; GRUDZINSKI, R. "Introdução: hegemonia biomédica e pluralismo ontológico no Brasil". In: MCCALLUM, A; ROHDEN, F. Corpo e saúde na Mira da Antropologia: ontologias, práticas, traduções. Salvador: EDUFBA/ABA, 2015. pp. 7-26.

MOL, A. "Care and its values: good food in the nursing home". In: MOL, A; MOSER, I; POLS, J. (eds.). Care in practice: on tinkering in clinics, homes and farms. Bielefeld: transcript Verlag, 2010.

MOL, Annemarie. The body multiple: ontology in medical practice. Durhan: Duke University Press, 2002.

MOREIRA, T; PALLADINO, P. Between truth and hope: on Parkinson's disease, neurotransplantation and the production of the 'self'. History of the Human Sciences, 18 (3): 55-82, 2005.

MOSER, I. Making Alzheimer's disease matter. Enacting, interfering and doing politics of nature. Geoforum, 39 (1): 98-110, 2008.

NEWPORT, Mary. Alzheimer's Disease: What If There Was a Cure? The Story of Ketones. Laguna Beach: Basic Health Publications, 2013.

NEWPORT, Mary. The Coconut Oil and Low-Carb Solution for Alzheimer's, Parkinson's, and Other Diseases: a guide to using diet and a high-energy food to protect and nourish the brain. Laguna Beach: Basic Health Publications, 2015. 
NEWPORT, Mary. The Complete Book of Ketones: A Practical Guide to Ketogenic Diets and Ketone Supplements. Laguna Beach: Basic Health Publications, 2019.

O'DONOVAN, O. et al. Tracking Transformations in Health Movement Organizations: Alzheimer's Disease Organizations and their Changing "Cause Regimes". Social Movement Studies, 12 (3): 316-334, 2013.

PINHEIRO, L; GONZALEZ, R.; FONTOURA, N. Expansão dos direitos das trabalhadoras domésticas no Brasil. Nota Técnica/IPEA, 10: 1-20, 2012.

SAFIOTTI, H. O trabalho da mulher no Brasil. Perspectivas, 5: 115-135, 1982.

SORJ, B.; FONTES, A. "O care como um regime estratificado: implicações de gênero e classe social”. In: HIRATA, H.; GUIMARÃES, N. (orgs.). Cuidado e Cuidadoras. As várias faces do trabalho de care. São Paulo: Atlas, 2012. pp. 103116.

TAYLOR, J. Engaging with Dementia: Moral Experiments in Art and Friendship. Culture, Medicine, and Psychiatry, 41 (2): 284-303, 2017.

ZELIZER, V. A. Economic Lives: How culture shapes the economy. Princeton: Princeton University Press, 2011. 


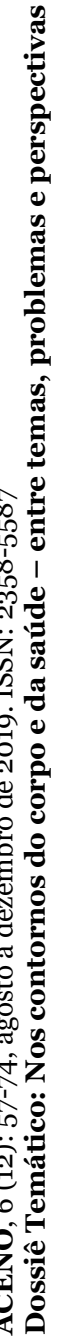

